\title{
Throughput Analysis for Cognitive Radio (CR) Systems
}

\author{
Ahmed S.Kadhim ${ }^{1}$ and Haider M. Alsabbagh ${ }^{2}$ \\ ${ }^{1}$ Department of Electrical Engineering, University of Basrah, Basrah, IRAQ \\ a.kadim@yahoo.com \\ ${ }^{1}$ Department of Electrical Engineering, University of Basrah, Basrah, IRAQ \\ haidermawdieee.com
}

\begin{abstract}
In cognitive radio networks, the secondary network (users) are allowed to utilize the frequency bands of primary network (users) when they are not currently being used. To support this function, the secondary users are required to sense the radio frequency environment, and once the primary user is found to be active, the secondary users have to vacate the channel within certain amount of time. There are two parameters related to channel sensing: probability of detection and probability of false alarm. The higher the detection probability, the better the primary users can be protected. However, from the secondary users' perspective, the lower the false alarm probability, the more chances the channel can be reused, thus the higher the achievable throughput for the secondary users. In this paper, we study the fundamental tradeoff between sensing capability and achievable throughput of the secondary users. Also, we present a design for sensing slot duration to maximize the achievable throughput for the secondary users under the constraint that the primary users are sufficiently protected. Using energy detection scheme, an optimal sensing time which provides the best tradeoff is given. Cooperative sensing is also studied based on the methodology of the proposed sensing throughput tradeoff. Computer simulations are presented to evaluate the proposed tradeoff methodology.
\end{abstract}

\section{KEYWORDS}

cognitive radio systems, throughput, wireless systems, wireless communications.

\section{INTRODUCTION}

Spectrum scarcity problem $[1,2]$, due to the growth of demand for the spectrum, is suggested to be solved by increasing the spectrum utilization which can be done by allowing cognitive users (unlicensed users) to occupy the spectrum band when the primary users (licensed users) do not use it. CR system [3,4] are suggested to use the spectrum band efficiently. One from different tasks which are performed by CR is the spectrum sensing [5] in which there are several sensing methods. Among of such methods is based on evaluating the energy detection $[6,7]$. Other methods were described in a numerous prestigious work, see for instance, Ref. [4, 8, 9]. Energy detection will be considered in this work due to its simplicity and no need for any prior information about the primary users' signals. Therefore, it has been thoroughly studied both in local spectrum sensing [6-10] and cooperative spectrum sensing [11-15]. In cooperative spectrum sensing, local spectrum sensing information from multiple CRs are combined for Primary User (PU) detection. In centralized CR network, a common receiver plays a key role in collecting these information and detecting spectrum holes which were described in details in [12]. 
International Journal of Computer Networks \& Communications (IJCNC) Vol.4, No.4, July 2012

In this paper, we analyze the effect of the probability of false alarm and the probability of the detection on the throughput of secondary networks. Star topology of the secondary network is considered, and the propagation time takes to collect the results of local sensing from each user as a delay. It is shown by the analyses that the throughput of the secondary decreases by cooperation delay which increases as the number of cooperation users increases.

This paper is organized as follows. Section 2 presents the system model for spectrum sensing. The network model and the method of cooperation is established in this section. In section 3, we study the theory about individual spectrum sensing and cooperative sensing based on energy detection. Section 4 studies the cooperation overhead for cooperative sensing. The throughput of secondary network is derived in

Section 5. The numeric results based on the analytical model are given in Section 6, and finally, conclusions are drawn in Section 7.

\section{SYSTEM MODEL}

This section briefly describes the analytical work to evaluate the performance of cognitive network where throughput and time sensing traffic are involved.

\subsection{Network model}

Cognitive users are distributed about distance radius $\mathrm{R}$ meters around the band manager. Let the density of secondary user (cognitive user) is A. then the number of cooperative user $\mathrm{I}$ is calculated by:

\subsection{Cooperation}

$$
\mathrm{I}=\mathrm{A} \cdot \pi \mathrm{R}^{2}
$$

In the cooperative sensing all cognitive users which exist within $R$ meter radius transmit their local decisions directly to the band manager via a control channel. Assuming transmission order is fixed beforehand, and secondary users transmit their results of sensing sequentially. Then the sum of the result of all terminals is compared with the threshold in the band manager. The judgment of whether primary signal is present or not is based on whether this sum passes the threshold or not. Cooperative sensing is achieved by broadcasting the judgment result from the band manager to all users.

\subsection{Spectrum Sensing}

This section presents, a survey on cooperative spectrum sensing for cognitive radio networks. A review of some well-know spectrum sensing techniques are presented and the principle of cooperative spectrum sensing is introduced. In order to avoid the harmful interference to the primary system, the cognitive radio needs to sense the availability of the spectrum. The goal of spectrum sensing is to decide between the following two hypotheses:

$$
\begin{aligned}
H_{0}: y(n) & =w(n), \\
H_{1}: y(n) & =w(n)+s(n), \\
n & =1, \ldots \ldots, N
\end{aligned}
$$


International Journal of Computer Networks \& Communications (IJCNC) Vol.4, No.4, July 2012

Where $\mathrm{y}(\mathrm{n})$ is received signal at the secondary user, $s(n)$ is transmitted signal from the primary user with the mean $\mu_{s}=0$ and the variance $\sigma_{s}^{2}, w(n)$ is an additive white Gaussian noise (AWGN) with the mean $\mu_{w}=0$ and the variance $\sigma_{w}^{2}$ and $N$ denotes the number of samples.

\subsection{Local Sensing}

We first consider local spectrum sensing at individual secondary users. The test statistic using energy detection is given

$$
\mathbf{T}=\sum_{\mathbf{n}=1}^{\mathbf{N}}|\mathbf{y}(\mathbf{n})|^{2}
$$

If the number of samples $N$ is large enough, we can use the central limit theorem to approximate the test statistic as Gaussian.

$$
\begin{gathered}
T \mid H_{o} \sim N\left(N \sigma_{w}^{2}, 2 N \sigma_{w}^{4}\right), \\
T \mid H_{1} \sim N\left(N\left(\sigma_{s}^{2}+\sigma_{w}^{2}\right), 2 N\left(\sigma_{s}^{2}+\sigma_{w}^{2}\right)^{2}\right)
\end{gathered}
$$

Where $\mathbf{N}(\boldsymbol{\mu}, \boldsymbol{\sigma})$ denotes the Gaussian distribution with the mean $\mu$ and the variance $\sigma$. Then the false alarm probability $P f$ and the detection probability $P d$ can be evaluated as

$$
\begin{aligned}
& P_{f}=Q\left(\frac{\gamma-N \sigma_{w}^{2}}{\sqrt{2 N \sigma_{w}^{4}}}\right) \\
& P_{d}=Q\left(\frac{\gamma-N\left(\sigma_{s}^{2}+\sigma_{w}^{2}\right)}{\sqrt{2 N\left(\sigma_{s}^{2}+\sigma_{w}^{2}\right)^{2}}}\right)
\end{aligned}
$$

Where $\boldsymbol{\gamma}$ denotes the threshold. If $\mathbf{P}_{\mathbf{f}}$ is given, $\boldsymbol{\gamma}$ can be determined from (8) by

$$
\gamma=\sqrt{2 \mathrm{~N} \sigma_{\mathrm{w}}^{4}} \mathbf{Q}^{-1}\left(\mathbf{P}_{\mathrm{f}}\right)+\mathrm{N} \boldsymbol{\sigma}_{\mathrm{w}}^{2}
$$

For a given pair of target probabilities $\left(\boldsymbol{P}_{\boldsymbol{F}}, \boldsymbol{P}_{\boldsymbol{D}}\right)$, the number of required samples to achieve these targets is related to the signal to noise ratio $S N R=\sigma_{s}^{2} / \sigma_{w}^{2}$ as follows:

$$
\mathbf{N}=2\left[\frac{\mathbf{Q}^{-1}\left(\mathbf{P}_{\mathrm{F}}\right)-\mathbf{Q}^{-1}\left(\mathbf{P}_{\mathrm{D}}\right) \cdot(\mathrm{SNR}+\mathbf{1})}{\mathrm{SNR}}\right]^{2}
$$




\subsection{Cooperative Sensing}

In the Cooperative Sensing, the local decisions from I secondary users are collected at a band manager via a dedicated control channel. The test statistic for Cooperative Sensing is given by summation of each $T$ as follows:

$$
T=\sum_{i=1}^{I} \sum_{n=1}^{N}\left|y_{i}(n)\right|^{2}
$$

In this paper, we assume the values of SNR of secondary users are same, namely $\sigma_{s, i}^{2}=\sigma_{s}^{2} \forall_{i}$, $\sigma_{w, i}^{2}=\sigma_{w}^{2} \forall_{i}$. In this case, to use the central limit theorem, we can approximate the test statistic as Gaussian.

$$
\mathrm{T} \mid \mathrm{H}_{\mathrm{o}} \sim \mathrm{N}\left(\mathrm{NI} \sigma_{\mathrm{w}}^{2}, 2 \mathrm{~N} \text { I } \sigma_{\mathrm{w}}^{4}\right)
$$

$$
\mathrm{T} \mid \mathrm{H}_{1} \sim \mathrm{N}\left(\mathrm{NI}\left(\sigma_{\mathrm{s}}^{2}+\sigma_{\mathrm{w}}^{2}\right), 2 \mathrm{NI}\left(\sigma_{\mathrm{s}}^{2}+\sigma_{\mathrm{w}}^{2}\right)^{2}\right)
$$

$\mathbf{P}_{\mathbf{f}}$ and $\mathbf{P}_{\mathbf{d}}$ can be evaluated as

$$
\begin{aligned}
& P_{f}=Q\left(\frac{\gamma-N I \sigma_{w}^{2}}{\sqrt{2 N I \sigma_{w}^{4}}}\right) \\
& P_{d}=Q\left(\frac{\gamma-N I\left(\sigma_{s}^{2}+\sigma_{w}^{2}\right)}{\sqrt{2 N I\left(\sigma_{s}^{2}+\sigma_{w}^{2}\right)^{2}}}\right)
\end{aligned}
$$

The threshold $\gamma$ is determined by given $\mathrm{P}_{\mathrm{f}}$ as

$$
\gamma=\sqrt{2 N I \sigma_{w}^{4}} Q^{-1}\left(P_{f}\right)+N I \sigma_{w}^{2}
$$

If we assume the minimum numbers of samples to achieve desired $\mathrm{P}_{\mathrm{F}}$ and $\mathrm{P}_{\mathrm{D}}$ is evaluated by

$$
N=\frac{2}{I}\left[\frac{Q^{-1}\left(P_{F}\right)-Q^{-1}\left(P_{D}\right) \cdot(S N R+1)}{S N R}\right]^{2}
$$


International Journal of Computer Networks \& Communications (IJCNC) Vol.4, No.4, July 2012

\subsection{The cooperation overhead:}

The cooperation overhead generally increases with the number of cooperating users due to the increased volume of data that needs to be reported to and be (centrally) processed by the band manager. Therefore, there exists a trade-off between the local processing overhead and the cooperation overhead as they both add to the total sensing time T. In this paper, we consider $\mathrm{T}$ as the addition of local sensing time Ts and the cooperation overhead Tc. Therefore the total sensing time $\mathrm{T}$ is evaluated by

$$
\mathbf{T}=\mathbf{T}_{\mathbf{c}}+\mathbf{T}_{\mathbf{s}}
$$

The local sensing time $T_{S}$ is given by:

$$
\begin{aligned}
& T_{s}=t_{s} N \\
& T_{s}=\frac{2 t_{s}}{I} \cdot\left[\frac{Q^{-1}\left(P_{F}\right)-Q^{-1}\left(P_{D}\right) \cdot(S N R+1)}{S N R}\right]^{2}
\end{aligned}
$$

Where $t_{s}$ is a sampling interval. Note that $T_{s}$ is the minimum sensing time to achieve the target probabilities $\mathrm{P}_{\mathrm{F}}$ and $\mathrm{P}_{\mathrm{D}}$.

Since the number of user $I$ is evaluated as shown in Equation (1), $T_{S}$ is expressed as a function of radius $R$ as follows:

$$
T_{s}=\frac{2 t_{s}}{A \pi} \cdot\left[\frac{Q^{-1}\left(P_{F}\right)-Q^{-1}\left(P_{D}\right) \cdot(S N R+1)}{R \cdot S N R}\right]^{2}
$$

In this paper, we consider only propagation time used to report the local decisions to band manager as cooperation overhead. We consider that secondary users identically distributed around the band manager. Therefore, the average distance from band manager to secondary user can be evaluated as $/ \sqrt{2}$. Then the average cooperation overhead per user is given by:

$$
\overline{\mathbf{T}}_{\mathbf{c}}=\frac{\mathbf{R}}{\sqrt{2} \mathrm{C}}
$$

where $C$ is the speed of light. Therefore, the cooperation overhead of secondary network is evaluated as

$$
\mathbf{T}_{\mathbf{c}}=\mathbf{I} \overline{\mathbf{T}}_{\mathbf{c}}
$$


International Journal of Computer Networks \& Communications (IJCNC) Vol.4, No.4, July 2012

$\mathbf{T}_{\mathbf{c}}=\mathbf{I} \cdot \frac{\mathbf{R}}{\sqrt{\mathbf{2}} \mathbf{C}}$

$$
\mathbf{T}_{c}=\frac{\mathbf{A} \pi \mathbf{R}^{3}}{\sqrt{2} \mathrm{C}}
$$

Since number of user I is evaluated as (1), $T_{s}$ decreases as number of users I increases, and $T_{c}$ increases as I increases.

From (19), total sensing time $T$ can be expressed as

$$
T=\frac{2 t_{s}}{A \pi} \cdot\left[\frac{Q^{-1}\left(P_{F}\right)-Q^{-1}\left(P_{D}\right) \cdot(S N R+1)}{R \cdot S N R}\right]^{2}+\frac{A \pi R^{3}}{\sqrt{2} C}
$$

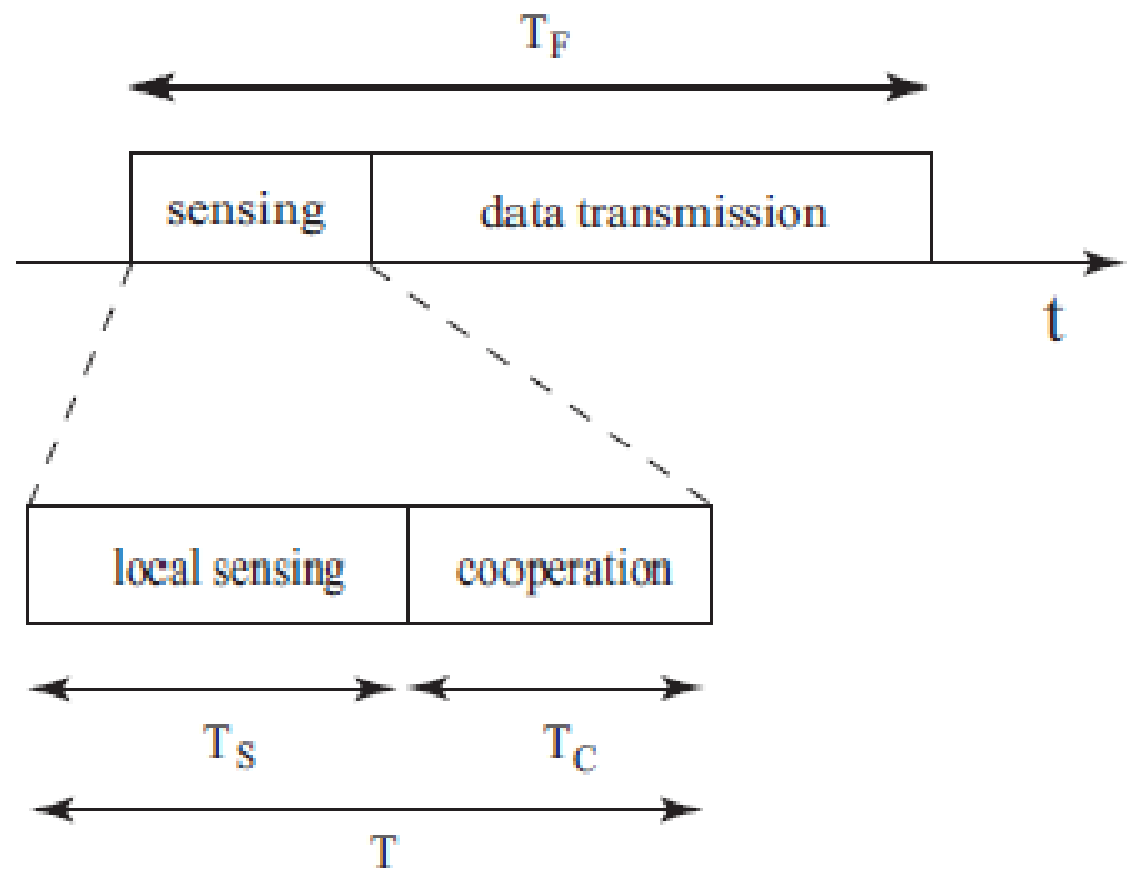

Figure 1. Communication frame for secondary networks 
International Journal of Computer Networks \& Communications (IJCNC) Vol.4, No.4, July 2012

\section{THE THROUGHPUT OF SECONDARY NETWORKS}

In this section, we analyze the effect of the total sensing time on the throughput of the secondary wireless system. Fig. 1 shows the communication frame of a secondary system assumed in this paper.

In each frame of duration $T_{F}$, secondary users sense the channel for a duration of $T_{S}$ given by (22). Then, secondary users transmit their local decisions to band manager for a duration of $T_{c}$. If none of the primary users is detected in the channel of interest, the secondary users will use the rest of frame $T_{F}-\left(T_{S}+T_{C}\right)$ for data transmission. If an active primary user is detected, secondary users will not transmit in this frame and wait until the next frame where the sensing of channel is repeated.

In this paper, we are interested in the communication of the secondary network that was able to be performed without causing the packet collision with the primary network.

When we assume the length of the communication frame of primary system as $\mathbf{T}_{\mathbf{P R F}}$, we consider the throughput of secondary network to the data that was able to be transmitted when primary network is not communicating during $\mathbf{T}_{\mathbf{P R F}}+\mathbf{T}_{\mathbf{F}}$.

Let us define $\mathbf{P}_{\mathbf{c}}$ as the probability that a primary user does not start transmission during $\mathbf{T}_{\mathbf{P R F}}+$ $\mathbf{T}_{\mathbf{F}}$. The normalized throughput of secondary network can then be expressed as

$$
\mathbf{T h}=\frac{\mathbf{T}_{\mathbf{F}}-\mathbf{T}}{\mathbf{T}_{\mathbf{F}}}\left(\mathbf{1}-\mathbf{P}_{\mathbf{f}}\right) \mathbf{P}_{\mathbf{c}}
$$

When the primary users have an exponential on-off traffic model, with the mean durations of on periods denoted by $\beta, P_{c}$ is given by:

$$
\mathbf{P}_{\mathrm{c}}=\exp \left(-\frac{\mathbf{T}_{\mathrm{PRF}}+\mathbf{T}_{\mathrm{F}}}{\beta}\right)
$$

Assuming that the frame of duration $\mathrm{T}_{\mathrm{PRF}}, \mathrm{T}_{\mathrm{F}}$ and the false alarm probability $P_{f}$ has been fixed, the normalized throughput of secondary network is given by:

$$
T h=\frac{T_{F}-T}{T_{F}}\left(1-P_{f}\right) \exp \left(-\frac{T_{P R F}+T_{F}}{\beta}\right)
$$

\section{RESULTS AND DISCUSSIONS}

Fig. 2 and Fig. 3 illustrate the relation between the local sensing time and the probability of false alarm and probability of detection respectively. Fig. 2 shows that at $S N R=-15 d B$ the local sensing time have the maximum value $(0.1619 \mathrm{msec})$ at probability of false alarm zero and Ts decreases as Pf increases until Pf become 0.9 the local sensing time start increasing again. at $\mathrm{SNR}=-10 \mathrm{~dB}$ the maximum value of local sensing time is(under $0.0184 \mathrm{msec}$ ) and still decreases until Pf become (0.9), Ts starting increase again. The best value of Ts is at SNR=-10dB.Fig. 3 shows that the inverse of the relation between Ts versus Pf. The main parameters values used in the model are that in [13] and arranged in Table 1. 
International Journal of Computer Networks \& Communications (IJCNC) Vol.4, No.4, July 2012

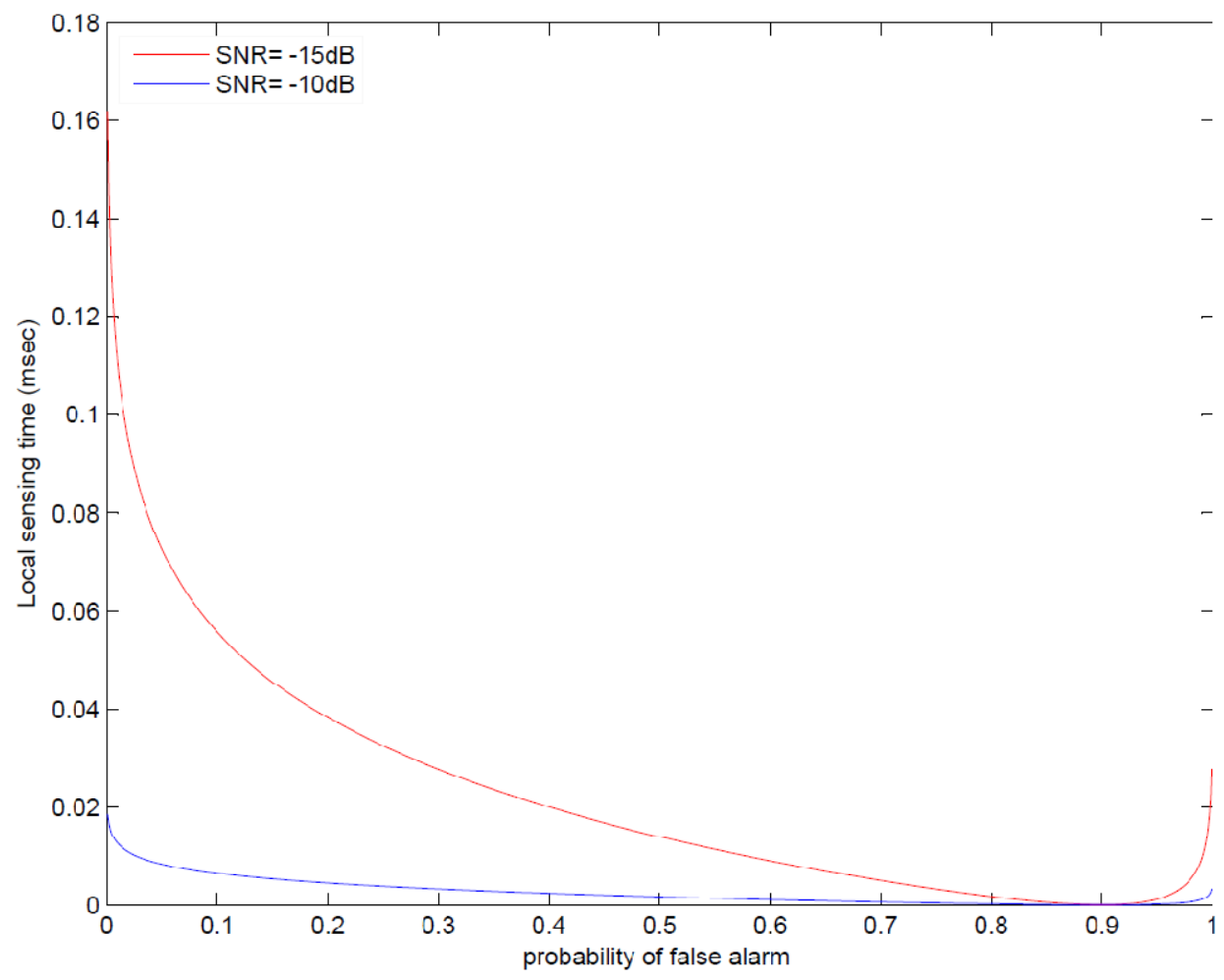

Figure 2. Local sensing time versus probability of false alarm at $S N R=[-15 d B-10 \mathrm{~dB}]$

Table 1. values of the used parameters

\begin{tabular}{|c|c|}
\hline Density of users (A) & $0.05 m^{-2}$ \\
\hline Cooperation radius $(\mathrm{R})$ & $40 \mathrm{~m}$ \\
\hline Probability of detection $\left(\mathrm{P}_{\mathrm{D}}\right)$ & 0.9 \\
\hline Probability of false alarm $\left(\mathrm{P}_{\mathrm{F}}\right)$ & 0.1 \\
\hline Length of the frame of secondary networks $\left(\mathrm{T}_{\mathrm{F}}\right)$ & $100 \mathrm{msec}$ \\
\hline $\begin{array}{l}\text { Length of communication frame of primary system } \\
\qquad\left(\mathrm{T}_{\mathrm{PRF}}\right)\end{array}$ & $150 \mathrm{msec}$ \\
\hline Speed of light (c) & $3 * 10^{8} \mathrm{~m} / \mathrm{sec}$ \\
\hline
\end{tabular}


International Journal of Computer Networks \& Communications (IJCNC) Vol.4, No.4, July 2012

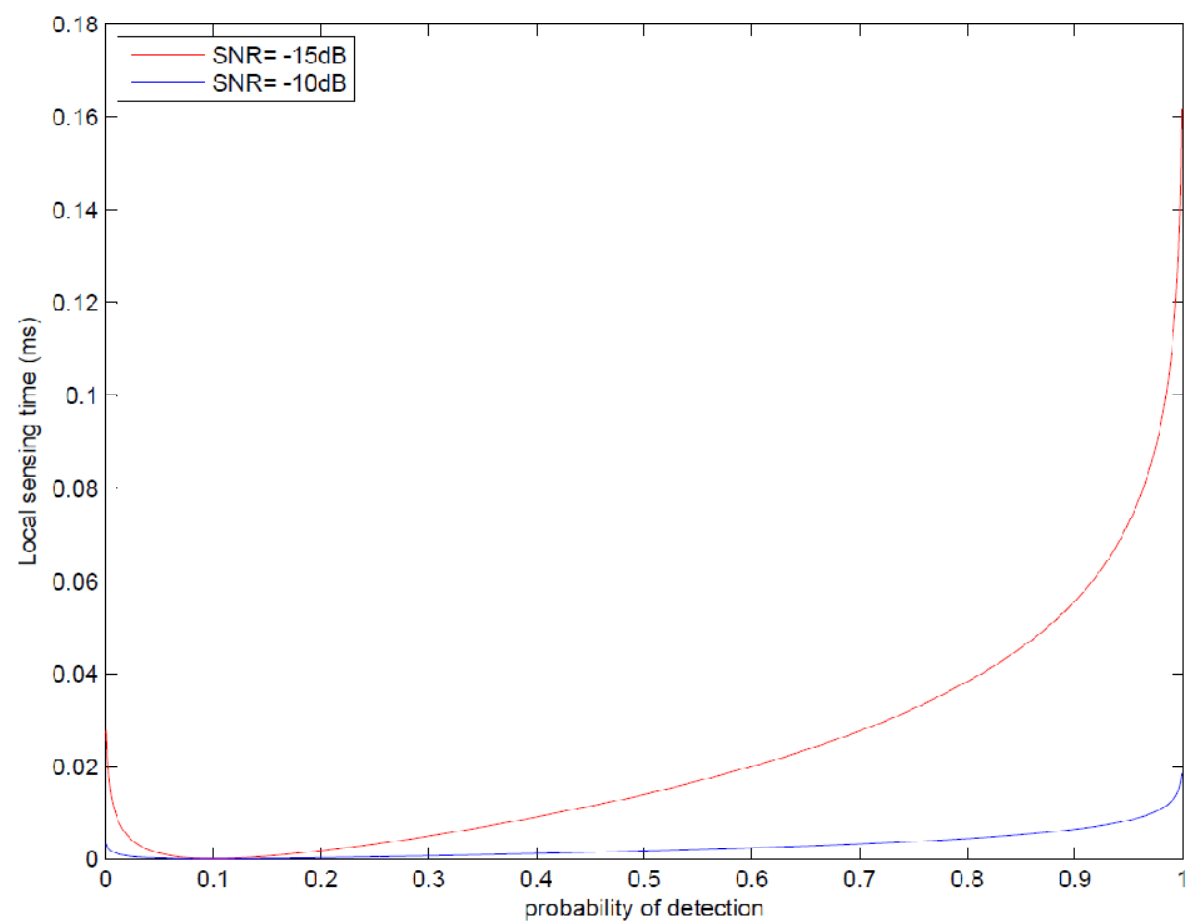

Figure 3. Local sensing time versus probability of detection at $S N R=[-15 \mathrm{~dB}-10 \mathrm{~dB}]$.

Fig. 4 and Fig. 5 illustrate the relation between the total sensing time and the probability of false alarm and probability of detection respectively at $S N R=-20 \mathrm{~dB}$. Fig. 4 the total sensing time have the maximum value $(1.575 \mathrm{msec})$ at probability of false alarm $(0.001)$ and $\mathrm{T}$ decreases as $\mathrm{Pf}$ increases until Pf become 0.9 the total sensing time start increasing again. Fig. 5 shows that the inverse of the relation between Ts versus Pf.

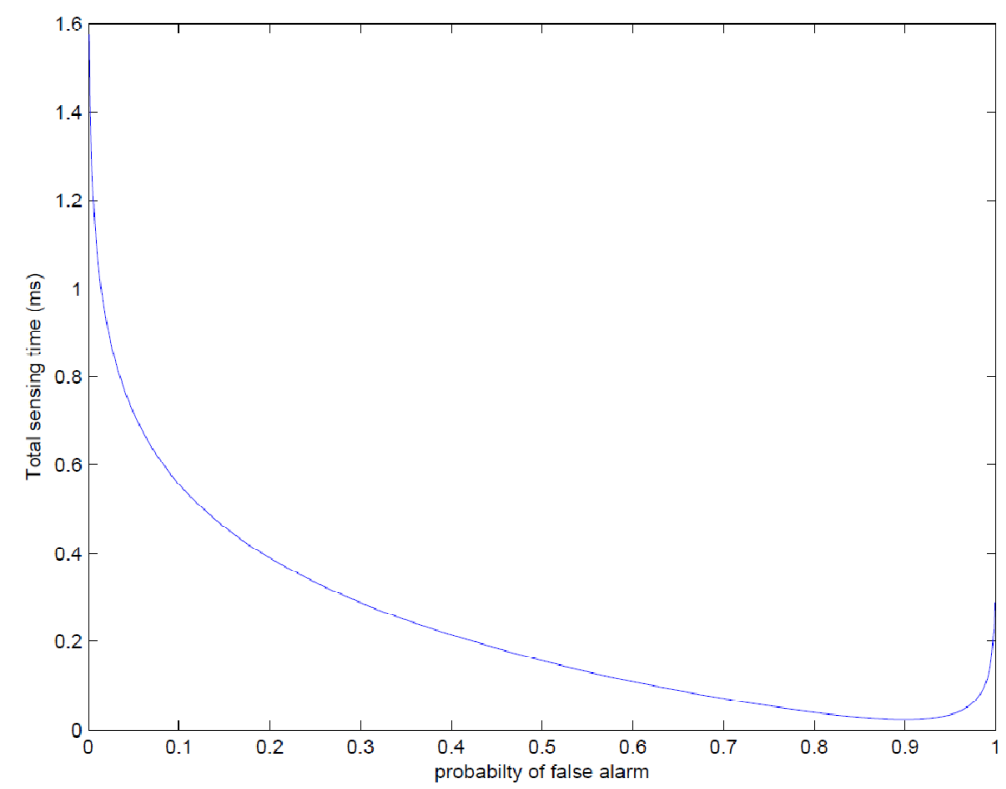

Figure 4. Total sensing time versus probability of false alarm at $\mathrm{SNR}=-20 \mathrm{~dB}$. 
International Journal of Computer Networks \& Communications (IJCNC) Vol.4, No.4, July 2012

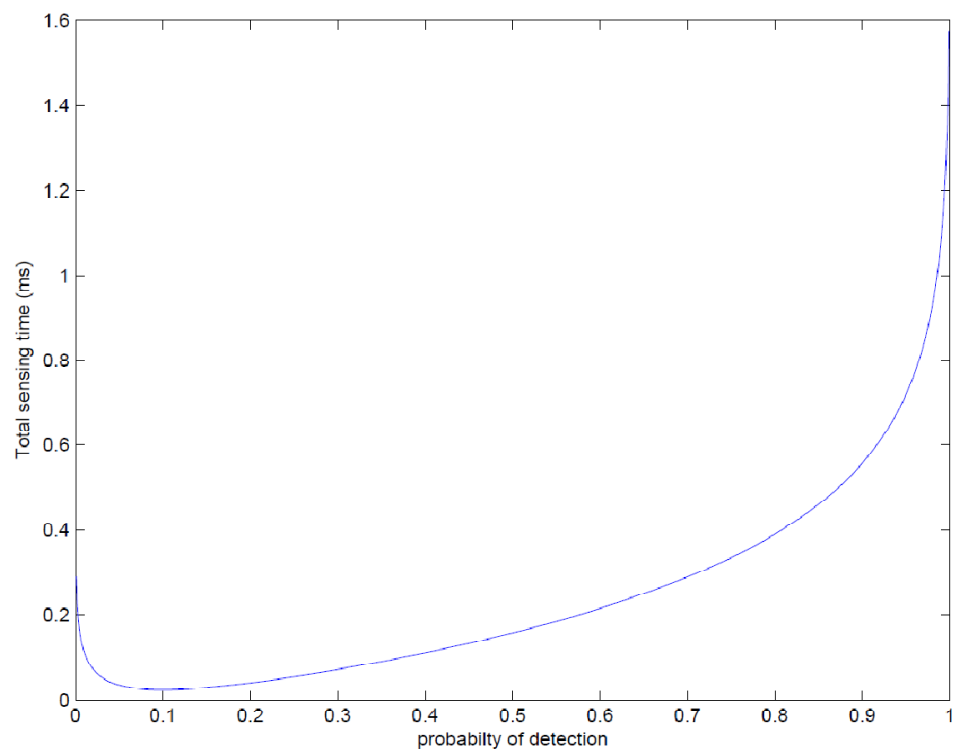

Figure 5: Total sensing time versus probability of detection at $\mathrm{SNR}=-20 \mathrm{~dB}$.

Figure 6 illustrates the throughput in the network as the probability of false alarm increases, for average interval between two starting time of communication of primary network: $\beta=(500450$ $400 \mathrm{msec}$ ). It is shown that for any values of $\beta$ the throughput decreases gradually when the probability of false alarm increases. The result means that the throughput of secondary network decreases due to the effect of the cooperation overhead.

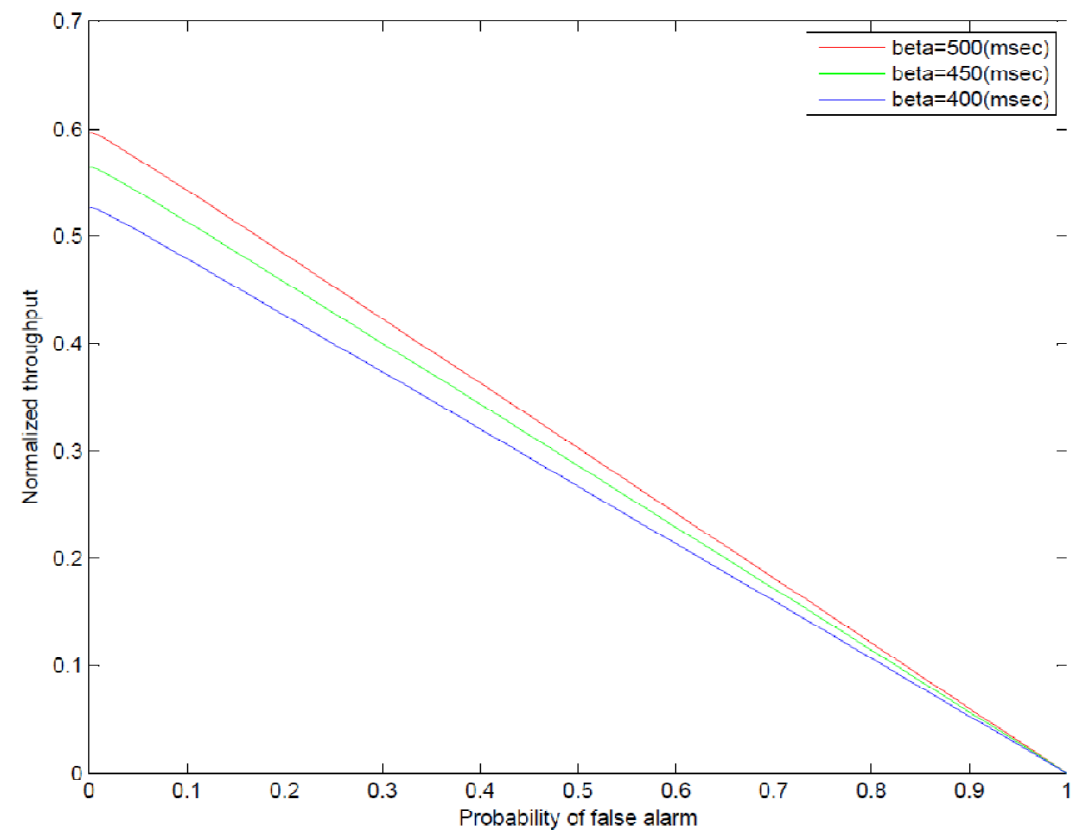

Figure 6. Normalized throughput for the secondary network versus probability of false alarm at $\mathrm{SNR}=-20 \mathrm{~dB}$. 
Figure 7 illustrates the throughput for the secondary network versus the probability of detection increases, for average interval between two starting time of communication of primary network: $\beta=(500450400) \mathrm{msec}$. It is shown that for any values of $\beta$ the throughput decreases gradually when the probability of detection increases. The result means that the throughput of secondary network decreases due to the effect of the cooperation overhead.

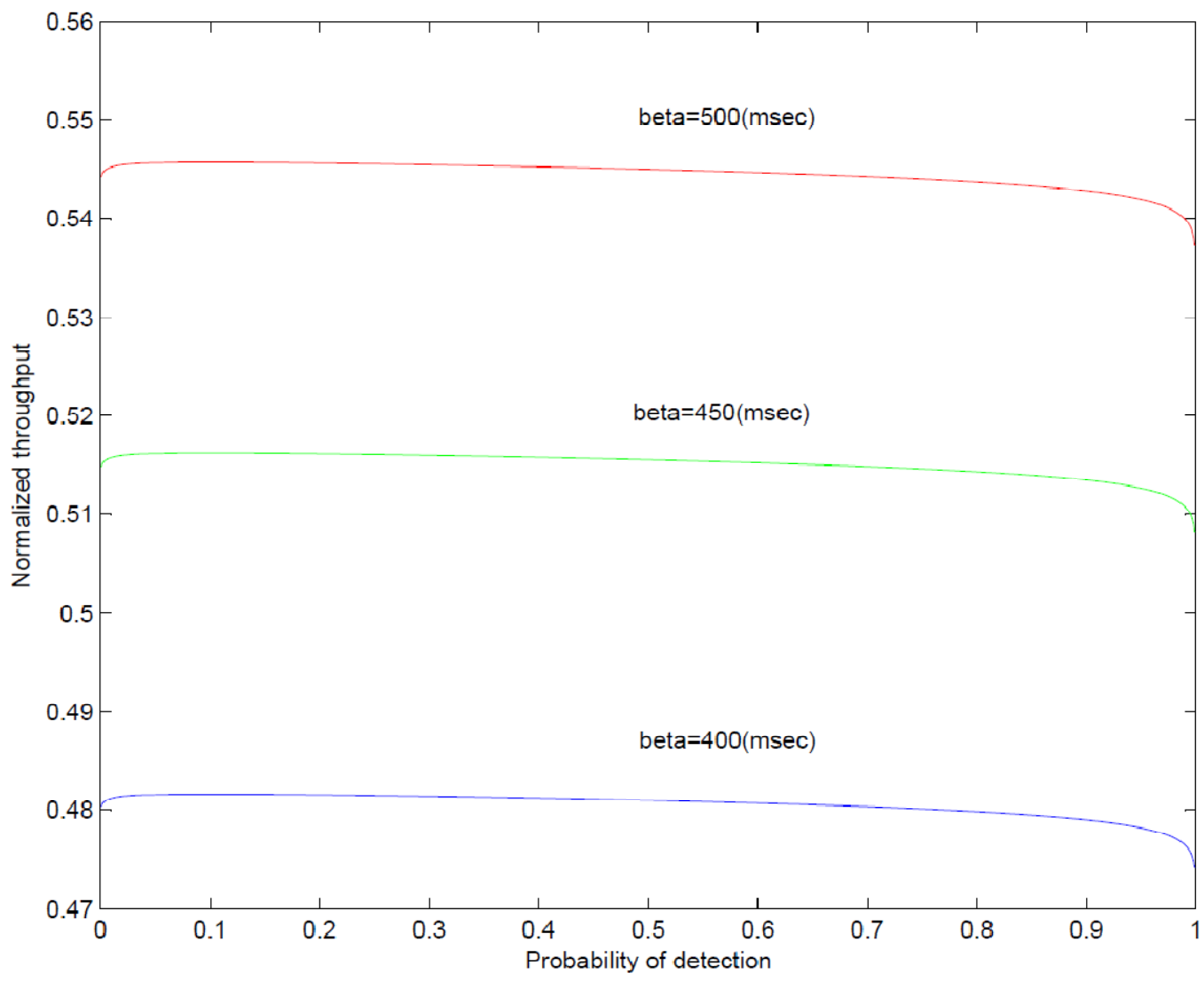

Figure 7. Normalized throughput for the secondary network versus probability of detection at $\mathrm{SNR}=-20 \mathrm{~dB}$.

\section{CONCLUSIONS}

In this paper, the effect of the cooperation overhead on the throughput of secondary network has been observed. Assuming propagation delay as the rudimentary cooperation delay, we have analyzed the performance of the secondary network. Total sensing time necessary to fill a specific detection property (target probability of detection and false alarm) and the throughput of secondary network has been derived. Numerical results have shown that the agility of sensing decrease due to increase in cooperation overhead as the number of cooperation users increases. Also, the throughput decreases gradually due to the decrease of agility as the cooperation overhead increases. In a cognitive radio network, the secondary users are allowed to utilize the frequency bands of primary users when these bands are not currently being used. To support this spectrum reuse functionality, the secondary users are required to sense the radio frequency environment, and once the primary users are found to be active, the secondary users are required to vacate the channel within a certain amount of time. Therefore, spectrum sensing is of significant importance in cognitive radio networks. There are two parameters associated with 
International Journal of Computer Networks \& Communications (IJCNC) Vol.4, No.4, July 2012

spectrum sensing: probability of detection and probability of false alarm. The higher the probability of detection, the better the primary users are protected. However, from the secondary users' perspective, the lower the probability of false alarm, the more chances the channel can be reused when it is available, thus the higher the achievable throughput for the secondary network.

\section{References:}

[1] Federal Communications Commission, "Spectrum Policy Task Force Report," FCC Document ET Docket No. 02-155, Nov. 2002.

[2] Notice of Proposed Rulemaking on Cognitive Radio, Federal Communications Commission (FCC) Std.No.03-322, Dec.2003.

[3] J. Mitola , "Cognitive Radio: An Integrated Agent Architecture for Software Defined Radio," Ph.D.Thesis, KTH, Stockholm, Sweden, 2000.

[4] S. Haykin, "Cognitive Radio: Brain-Empowered Wireless Communications," IEEE J. Select. Areas Commun., vol. 23, no. 2, pp. 201-220, Feb. 2005.

[5] A.Sahai and D.Cabric, "A Tutorial on Spectrum Sensing: Fundamental Limits and Practical Challenges," IEEE DySPAN2005, Baltimore, MD, Nov.2005.

[6] Kasun T. Hemachandra and Norman C. Beaulieu, " Novel Analysis for Performance Evaluation of Energy Detection of Unknown Deterministic Signals Using Dual Diversity," in Proc. Of IEEE, pp. 1$5,2011$.

[7] F. F. Digham, M. S. Alouini, and M. K. Simon, "On the Energy Detection of Unknown Signals over Fading Channels," IEEE Transactions on Communications, vol. 55, no. 1, Jan. 2007.

[8] D. Cabric, S.M. Mishra, and R.W. Brodersen, "Implementation Issues in Spectrum Sensing for Cognitive Radios". IEEE Asilomar Con! Signals, Systems and Computers, vol. 1, pp. 772-776, Nov. 2004.

[9] H. Tang, " Some Physical Layer Issues of Wide-Band Cognitive Radio Systems". IEEE Int. Con! Wireless Networks, Commun. and Mobile Computing, pp. 151-159, Nov. 2005.

[10] R. Tandra and A. Sahai, "Fundamental Limits on Detection in Low SNR under Noise Uncertainty," IEEE Int. Con! Wireless Networks, Commun. and Mobile Computing, vol. 1, pp. 464-469, June 2005.

[11] Geng Wang "Performance of Collaborative Spectrum Sensing in a Cognitive Radio System" Master Thesis, The University of British Columbia, 2009.

[12] Khaled Ben Letaief, and Wei Zhang, "Cooperative Communications for Cognitive Radio Networks " Proceedings of the IEEE, vol. 97 , Issue: 5, May 2009.

[13] Junyang Shen, Tao Jiang, Siyang Liu and Zhongshan Zhang, "Maximum channel throughput via cooperative spectrum sensing in cognitive radio network" proceedings of the IEEE, vol. 8, Issue: 10, pp. 5166-5175, Oct. 2009.

[14] Song Jingqun, Feng Zhiyong, Fan Dian, Xue Jiantao, Liu Zemin, " Maximum Channel Throughput via Cooperative Spectrum Sensing in Cognitive Radio Networks," IEEE Int. Con! Wireless Communication and Networking, pp. 1-5, 2010.

[15] Shuquan Xia and Lei Zhang, " Optimization for Cooperative Spectrum Sensing in Cognitive Radio Networks," IEEE Int. Con! Intelligent Information Technology Application, vol. 3, pp. 331-334, 2010. 